\title{
Tiempo libre: una fuente de vida en la educación
}

\author{
Free time: a source of life in education \\ Tempo livre: uma fonte de vida na educação
}

\begin{abstract}
Ángel Miller Roa Cruz
Doctor en ocio, cultura y comunicación para el desarrollo humano Universidad Surcolombiana miler.roa@usco.edu.co

Kelly Julieth Sánchez Arboleda Estudiante de licenciatura en pedagogía infantil Universidad Surcolombiana kellyjuliethsanchezarboleda@gmailcom
\end{abstract}

\section{Resumen}

Se presentan los resultados de una investigación cuantitativa para determinar el uso que le dan los docentes al tiempo libre. La población objeto de estudio estuvo conformada por los 7 programas de educación con un total de 82 docentes de planta de la Universidad Surcolombiana, con el fin de determinar su interacción con el medio y la manera cómo perciben la calidad de vida y el uso del tiempo libre en su cotidianidad, por medio de una actividad lúdico recreativa con su núcleo familiar. Finalmente se concluye que los docentes de planta al tener tanta carga laboral dejan a un lado ese tiempo libre para recrearse, dando prioridad a las obligaciones académicas.

Palabras clave: Actividades ludo recreativas, tiempo libre, ocio, calidad de vida.

\begin{abstract}
The results of a quantitative investigation are presented to determine the use that teachers give to free time. The population studied was made up of the 7 education programs with a total of 82 teachers of the Surcolombiana University, in order to determine their interaction with the environment and how they perceive the quality of life and the use of free time in their daily lives, through a recreational activity with their family nucleus. Finally, it is concluded that the teachers of the plant, having so much work load leave aside that free time to recreate, giving priority to the academic obligations.
\end{abstract}


Keywords: Leisure activities, leisure time, leisure, quality of life.

\section{Resumo}

Os resultados de uma investigação quantitativa são apresentados para determinar o uso que os professores dão ao tempo livre. A população estudada foi composta pelos 7 programas de educação com um total de 82 professores da Universidade Surcolombiana, a fim de determinar sua interação com o ambiente e como eles percebem a qualidade de vida e o uso do tempo livre em suas vidas diárias, através de uma atividade recreativa com o núcleo familiar. Por fim, conclui-se que os professores da fábrica, tendo tanto trabalho de carga deixam de lado aquele tempo livre para recriar, priorizando as obrigações acadêmicas.

Palavras-chave: atividades ludo recreativas, tempo livre, tempo, qualidade de vida.

\section{Introducción}

Según lo planteado en el artículo 117 de la ley 30, las instituciones de educación superior deben adelantar programas de bienestar, entendidos éstos como el conjunto de actividades que se orientan al desarrollo físico, psicoafectiva, espiritual y social de los docentes. En este sentido determinar el uso que le dan los docentes al tiempo libre, de forma personal, social y familiar desde la óptica de la persona, nos permite crear estrategias para conocer y contribuir al aprovechamiento de este tiempo tan valioso de los docentes de planta de la facultad de educación en la Universidad Surcolombiana.

Las diferentes actividades realizadas fuera del ámbito laboral de las profesiones $u$ oficios se están viendo afectadas hoy en día por la "falta de tiempo ", el tiempo laboral que no se maneja adecuadamente se convierte en una burbuja que encierra $y$ suprime la vida del ser humano, ahora bien, en la Universidad Surcolombiana se quiere implementar un nuevo proyecto en el cual los docentes de planta de la facultad de educación cuenten con el privilegio de tener momentos recreativos con sus familiares en su tiempo libre y así despejarse un poco de sus cargas académicas y dedicar ese tiempo a ellos mismos $\mathrm{y}$ a sus familias.

En la Universidad Surcolombiana, el proyecto tiempo libre: una fuente de vida en la educación, se crea con el fin de contribuir a resolver interrogantes sobre las problemáticas y necesidades que hacen parte de la vida personal, familiar y laboral de los maestros de planta de la facultad de educación, en torno al uso y aprovechamiento que le dan al tiempo libre, a la vez de brindar pautas y estrategias pedagógicas 
que contribuyan a mejorar calidad de vida de cada uno de los docentes. Al involucrar la recreación y la actividad física en los docentes de la facultad de educación es evidente que el aprendizaje que se adquiere se hace más activo, a su vez se establecen relaciones con capacidades ya adquiridas y tratan de adaptarlas a las condiciones espacio-temporales que surgen en su exterior, también exploran esquemas nuevos que se presentan como respuesta a las diferentes situaciones-problemas derivados del juego enfrentándose a una situación en donde escuchan diferentes opiniones de sus compañeros docentes y se busca una solución.

\section{Referentes teóricos}

De acuerdo con lo anterior, las investigaciones realizadas en otros contextos permiten tomar ciertos aportes y referencias que son muy importantes y de soporte en este proyecto para despejar dudas y aclarar significados para un nuevo conocimiento.

El tiempo libre responde a "las formas personales como cada individuo organiza su vida cotidiana", es decir "la forma original individualizada, no solo de particularidades en cuanto al contenido de sus creencias, valores o formas de comportamiento cotidiano, sino a la manera como cada persona vive las normas de su grupo, clase y sociedad a la que pertenece" (Valdez, 2000).

Por su parte (Moreschi, 2000) en una investigación plantea que el "tiempo libre, en nuestra concepción, es un tiempo que se desarrolla en un marco donde la libertad no sufre condicionamientos externos lo suficientemente fuertes como para no permitirnos un desarrollo vital realmente libre".

La Universidad Surcolombiana a través del grupo de investigación MOLUFODE, su semillero OTIUM del programa de Educación Física y con el apoyo permanente de jóvenes investigadores del programa, cuenta con personas que mediante la metodología de investigación crean soluciones viables a las problemáticas que se identifican en el uso y aprovechamiento del tiempo libre de la población objeto, contribuyendo de manera organizada, planificada y directa con actividades lúdicas, deportivas, recreativas y culturales.

Para Manuel Cuenca (2000): "Cuando decimos tiempo libre a menudo nos referimos a un ámbito temporal lleno de posibilidades, que depende de nosotros. Un tiempo en el que la ausencia de obligaciones nos permite llevar a cabo acciones de cualquier tipo. Decir tiempo de ocio es frecuente que nos remita al tiempo empleado específicamente en prácticas de 
ocio". Se debe tener en cuenta que el tiempo libre también es un momento que se emplea para descansar, dejando a un lado aquellas actividades laborales que al ser frecuentes en el diario vivir generan estrés o cansancio físico.

\section{Metodología}

El presente estudio comprende una población total de 82 docentes de planta de la Facultad de Educación de la Universidad Surcolombiana de los 7 programas que esta comprende, 14 docentes del programa de inglés, 6 docentes del programa de matemáticas, 7 docentes del programa de ciencias naturales, 9 docentes del programa de pedagogía, 12 docentes del programa de lengua castellana, 7 docentes del programa de artes, 12 docentes del programa de educación física y 15 docentes del departamento de psicopedagogía.

A todos los docentes de planta de la Facultad de Educación se les aplicó una encuesta de selección múltiple la cual fue elaborada por nuestro grupo de trabajo conformado por estudiantes pertenecientes al semillero de investigación OTIUM. En la actividad ludo recreativa realizada el 18 de noviembre del 2017 en el Club Comfamiliar Los Lagos de la ciudad de Neiva-Huila, se invitó a las familias de los docentes de la facultad de educación de la Universidad Surcolombiana a compartir una tarde ludorecreativa con sus familiares, donde tuvieron la oportunidad de experimentar diferentes tipos de juegos grupales, tales como calentamientos, actividades deportivas y recreativas donde se fomentaba la importancia del aprovechamiento del tiempo libre a nivel personal, familiar y social. La asistencia de los docentes en la actividad no fue la esperada por los organizadores, pues fueron pocos docentes con sus familias quienes participaron en esta. Finalmente, se le brindo a cada asistente un refrigerio nutritivo y se les aplicó la encuesta. Los docentes que no asistieron a la actividad diligenciaron la encuesta en su sitio de trabajo.

El programa de educación física, recreación y deporte por medio del semillero OTIUM, inicia con los estudiantes del proyecto de investigación el respectivo análisis de la encuesta aplicada partiendo de la problemática de esta población que se investiga, la cual es la ocupación de tiempo libre de los docentes de planta de la facultad de educación a nivel personal, social y familiar.

La encuesta sobre el uso del tiempo libre permite conocer indicadores que reflejan hábitos y estilos de vida de la población, así como mostrar la situación y comportamiento de los docentes dentro de la sociedad actual, en su ámbito laboral, familiar y personal. Con esta encuesta pudimos encontrar que los maestros utilizan el tiempo libre para estar 
en familia, leer, adelantar algunas labores de su trabajo, en su espiritualidad y religiosidad, hacer deporte los domingos mayormente y asistir a eventos o lugares donde haya esparcimiento, todo con un carácter comparativo respecto a estudios previos y otros posteriores, que permitan conocer la evolución de este tiempo en sus vidas, frente a las condiciones sociales actuales.

La información recolectada se organizó a partir de las categorías de análisis propuestas, una vez se estructuró la información, se sistematizó, teniendo en cuenta los criterios establecidos en cada pregunta.

\section{Resultados}

La gran mayoría de los docentes de planta de la Facultad de Educación comprenden por tiempo libre el concepto de Manuel Cuenca: "tiempo libre a menudo nos referimos a un ámbito temporal lleno de posibilidades, que depende de nosotros. Un tiempo en el que la ausencia de obligaciones nos permite llevar a cabo acciones de cualquier tipo", a su vez las actividades que los docentes realizan con más frecuencia en el tiempo libre es ver la televisión y leer pero muy pocos docentes utilizan este tiempo para recrearse. El 99\% de los docentes afirmaron la importancia de compartir el tiempo libre en familia frente al 1 \% que no lo creía importante, pues este tiempo siempre lo ha ocupado en sí mismo. Ahora bien, un aspecto a destacar es que el $73 \%$ de los docentes no conocen ningún programa de la Universidad Surcolombiana que promueva el uso y aprovechamiento del tiempo libre, lo que da paso a crear estrategias para solucionar esta problemática y así promover el buen uso de este tiempo.

La mayoría de los docentes, el $42 \%$, utilizan el tiempo libre para realizar actividades fuera de su ámbito laboral, una de estas es el ver televisión, el 41 \% de docentes leen en su tiempo libre, pero un porcentaje mínimo de $22 \%$ son los que se recrean en ese tiempo.

Otro aspecto importante a destacar es que el 98\% de los docentes de planta creen que el uso del tiempo libre es muy importante para el desarrollo personal y el 85\% dedican ese tiempo libre a sus familiares, a su vez para el 99\% de los docentes es significativo compartir este tiempo en familia a diferencia del 10\% quienes utilizan ese tiempo libre para compartir con sus amigos y un 3\% pasan el tiempo libre con la pareja sentimental mientras que un total de $2 \%$ prefieren pasar el tiempo libre solos.

Ahora bien, el 73\% de los docentes de planta no conocen las actividades que la universidad programa para el aprovechamiento del tiempo libre en familia, a diferencia del $27 \%$ de estos que si 
conocen actividades promovidas por la universidad pero muy pocas y por ende no participan.

Otra de las preguntas realizadas en la encuesta fue la frecuencia con la que el docente realiza actividades para el aprovechamiento del tiempo libre, ante esta pregunta, el $40 \%$ de los docentes semanalmente realizan diferentes actividades, el 28\% dicen que diariamente, $23 \%$ que ocasionalmente y el 9\% lo hace mensualmente.

Finalmente al $85 \%$ de los docentes encuestados les gustaría que el programa de educación física en coordinación con la facultad de educación programara los fines de semana actividades que promuevan el aprovechamiento del tiempo libre dirigidas a los docentes y su núcleo familiar.

Los resultados obtenidos por la investigación fueron socializados en el grupo del semillero Otium, dando paso a una propuesta de intervención para implementarla con los docentes de planta de la facultad de educación de la Universidad Surcolombiana.

\section{Propuestas y Conclusiones}

Se evidencia que el concepto de tiempo libre que realizan los docentes de planta de la facultad de educación es descansar más que realizar actividades recreativas que promueven una integración social con los demás docentes. El aprovechamiento del tiempo libre es muy importante para los docentes de planta de la facultad de educación.

A través de una propuesta ludo recreativa se quiere romper esquemas implantados y crear nuevas herramientas, metodologías que direccionen los hábitos del aprovechamiento del tiempo libre para los docentes de planta de la facultad de educación, con el fin de desarrollar actividades ludo recreativas y así establecer un cronograma de actividades en espacios planeados por el investigador para continuar con el proyecto y poder contribuir en el desarrollo del docente a nivel personal, familiar y social.

Se considera que los conceptos sobre recreación, ocio y tiempo libre, deben ser actuales, claros, generales para toda la población, permitiendo su comprensión y apropiación alcanzando diferentes sectores como el profesional, familiar, personal. Es prevalente que este tipo de investigaciones trasciendan a la práctica, donde su metodología sirva de base para establecer procesos de intervención en diferentes sectores poblacionales, convirtiéndose a futuro en un elemento base para el impacto que debe tener la recreación, el ocio y el tiempo libre a nivel local, regional, nacional e internacional. 


\section{Referencias}

Motta, F. (30 de junio de 2015). Razones para usar la bicicleta. (M. Mora, Ed.) Salud\&Guia familiar (9), 14.

Hurtado, C. A. (2008). Funlibre obtenido de centro de documentación virtual en recreación, tiempo libre y ocio: http://www.redcreacion.org/d ocumentos/congreso10/CHuer tas.html

Cuenca, M. (2000). Ocio Humanista, dimensiones y manifestaciones actuales del ocio. Recuperado el 25 de septiembre de 2016, de ocio humanista, dimensiones y manifestaciones actuales del ocio: http://www.deustopublicaciones.es/deusto/pdfs/ ocio/ocio16.pdf

Santamaría, M. (2000), Ocio, calidad de vida y discapacidad -capitulo 2; publicación de la cátedra de ocio y minusvalías con motivo del 6 Congreso Mundial de Ocio
Constitución política de Colombia de 1991, actualizada con los actos legislativos a 2015.

Moreschi, C. (Abril de 2000). www.google.com.co.Obtenido de www.google.com.co: http://www.lazer.eefd.ufrj.br/ producoes/aproximandonos_a _la_problematica_del_tiempo_l ibre.pdf

De Grazia, S. (1966) Tiempo, trabajo y Ocio, Madrid, Tecnos, p. XIX.

Salta, $\quad$ E. (2015). www.google.com.co.Recuperad o el 12 de septiembre de 2015, de

www.google.com.co:http://ww w.estilosalta.com/psico/44psicologia/1129-caprovecharnuestro-tiempo-libre.html

Valdez, D. (2.000) La ludoteca: una alternativa de educación para el uso del tiempo libre). Colofón. México, D.F. 


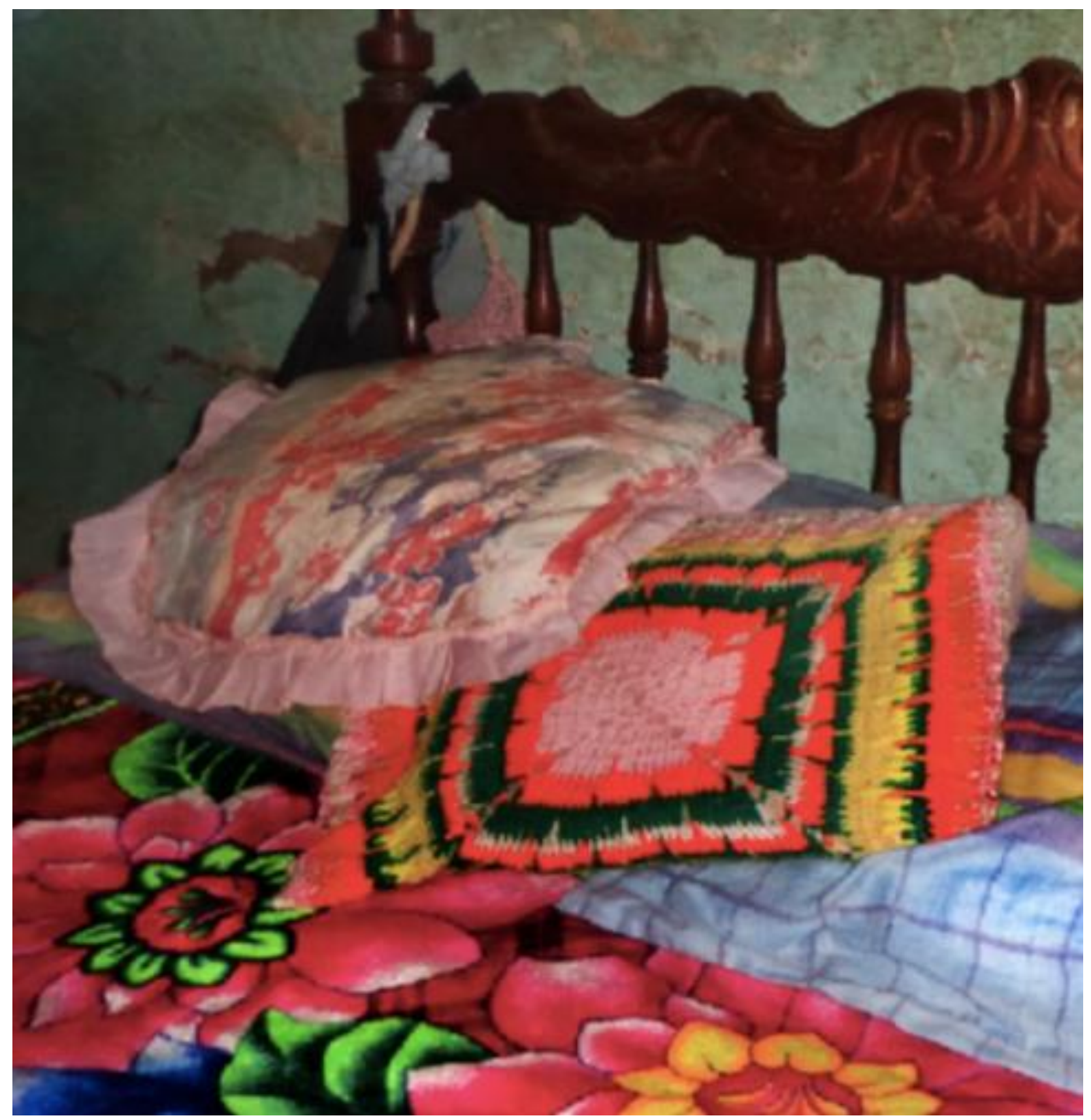

Número de la obra: 10

Título: "sobre camas"

Autor: Yenny Banesa Bonilla

Lugar: Zona rural municipio de Baraya

Finca: Los laureles

Fecha de captura: Marzo 2 de 2014

Técnica: Fotografía digital

Fuente: Bonilla, Y. B. (2014). Sobre camas. Una mirada estética popular del municipio de Baraya. Tesis de pregrado. Universidad Surcolombiana. Neiva, 2014. p.79. 